\title{
Control of the finite element discretization error during the convergence of structural shape optimization algorithms
}

\author{
Gabriel Bugeda ${ }^{1,3, a}$, Juan José Ródenas ${ }^{2}$, José Albelda ${ }^{2}$, Eugenio Oñate ${ }^{3}$ \\ ${ }^{1}$ Escola Universitària d'Enginyeria Tècnica Industrial de Barcelona (EUETIB), Universitat Politècnica de Catalunya (UPC), C/ Comte d'Urgell, \\ 187, 08036, Barcelona, Spain \\ ${ }^{2}$ Centro de Investigación de Tecnología de Vehículos (CITV), Universidad Politécnica de Valencia (UPV), Camino de Vera s/n, 46022, Valencia, \\ Spain \\ 3 International Center for Numerical Methods in Engineering (CIMNE), Universitat Politècnica de Catalunya (UPC), C/ Gran Capitán s/n, Cam- \\ pus Nord UPC, Módulo C1, 08034, Barcelona, Spain
}

Received 20 May 2009, Accepted 12 August 2009

\begin{abstract}
This work analyzes the influence of the discretization error contained in the Finite Element (FE) analyses of each design configuration proposed by structural shape optimization algorithms over the behaviour of the algorithm. If the FE analyses are not accurate enough, the final solution will neither be optimal nor satisfy the constraints. The need for the use of adaptive FE analysis techniques in shape optimum design will be shown. The paper also proposes the use of the algorithm described in [1] in order to reduce the computational cost associated to the adaptive FE analysis of each geometrical configuration when evolutive optimization algorithms are used.
\end{abstract}

Key words: Structural shape optimization, Adaptive remeshing, Sensitivity analysis, Evolutionary algorithms

\section{Introduction}

From a mathematical point of view, the treatment of an optimization problem can be viewed as the minimization of a function $f(\mathbf{x})$ depending on a set of variables $\mathbf{x}$ and subjected to some constraints. The general form of such a problem is:

$$
\begin{array}{ll}
\text { minimize : } & f(\mathbf{x}) ; \mathbf{x}=\left\{x_{i}\right\} ; i=1, \ldots, n \\
\text { with : } & \mathbf{g}(\mathbf{x})=\left\{g_{j}(\mathbf{x})\right\} ; j=1, \ldots, m \\
\text { verifying : } & g_{j}(\mathbf{x}) \leq 0 \quad ; j=1, \ldots, m \\
& a_{i} \leq x_{i} \leq b_{i} \quad ; i=1, \ldots, n
\end{array}
$$

where $f$ is the objective function (OF), $x_{i}$ are the design variables and $g_{j}$ are inequality constraints which, for structural problems, are normally expressed in terms of stresses and/or displacements. The values $a_{i}$ and $b_{i}$ define lateral constraints. Each individual is characterized by a set of values of $\mathbf{x}$ that correspond to a specific structural design. The definition of each design in terms of the values of $\mathbf{x}$ is called the parameterization of the optimization problem. The resolution of the optimum design problem consists of finding the values of $\mathbf{x}$ defining the best design.

The algorithms used to solve optimization problems are generally iterative. Whichever the algorithm used, it would be necessary to evaluate the values $f$ and $\mathbf{g}$ for each of the different designs during the iterative optimization process. In this work we have considered structural shape optimization. The values for $f$ and $\mathbf{g}$ in this kind of problems are usually obtained by the use of the finite element method (FEM). Hence, one should create a specific mesh for each of the different designs to be considered and then use the FEM to obtain the structural response of each design and, if necessary, the corresponding sensitivities with respect to the design variables. Two main aspects relative to the evalua- tion of $f$ and $\mathbf{g}$ by means of the FEM, which have a great importance over the global behavior of the optimization process, must be taken into account: the computational effort required for the numerical evaluation of each individual (geometrical configuration) and the accuracy of the FEM results.

The importance of the computational effort required for the evaluation of each geometrical configuration is evident. In optimization problems, like those under consideration, most of the computational cost is devoted to the analysis of individuals in order to obtain the values of the OF and the degree of satisfaction of the constraints.

On the other hand, and related to the accuracy of the results, we have to be aware that this numerical analysis techniques only provide approximate values for the data required by the optimization algorithm (OF and constraints). If these values are not accurate enough, an excessive amount of noise can be introduced in the optimization process. This could decrease the rate of convergence of the optimization process to the optimal solution, produce the convergence of the process to a non-optimal solution or, simply, avoid convergence. In the context of the FEM, the so called $h$ adaptive techniques, the $p$-adaptive techniques and the $h p$ adaptive techniques can be used to obtain solutions with the prescribed accuracy level. However, the use of these techniques implies a big computational cost that reduces the computational efficiency of the optimization process.

This paper shows that the correct behavior of the optimization process is only obtained if a minimum quality of the results of the analysis of each design, used to drive the optimization process, is ensured. To do this we will show the effect of the prescribed maximum error in energy norm over the final results obtained by means of a gradient-based deterministic algorithm and those obtained with an evolutionary algorithm.

${ }^{a}$ Corresponding author: bugeda@cimne.upc.edu 
As previously commented, adaptive FEM techniques can be used to obtain solutions with a prescribed accuracy level. However, in the traditional adaptive techniques this implies the successive analysis of numerical models with increasing accuracy, automatically adapted to the characteristics of the solution of the problem. The computational cost related to the use of these traditional adaptive analysis techniques can be critical when evolutionary algorithms are used. To solve this problem, in this paper we propose the use of the $h$-adaptive analysis technique for the individuals of each generation described in [1], that avoids the full $h$-adaptive remeshing loop for the vast majority of them. We also propose to increase the required level of accuracy in the FE analyses during the evolution of the optimization process in order to decrease the computational cost at the early stages of the process while ensuring the quality of the final results of the optimization.

\section{Case study and motivation. Pipe cross section}

Here we present the results of the solution of an optimization problem, with a known analytical solution, using different levels of the controlled discretization error, as a motivation for the developments presented in this paper. Both, evolutionary and deterministic optimization algorithms have been considered. The part to be optimized corresponds to the cross-section of a pipe subjected to an internal pressure. Two perpendicular planes of symmetry have been considered; therefore, only one quarter of the section has been modeled, as shown in Figure 1. The shape is defined by a total of 8 points, 5 of these points describe a circular internal shape and 3 describe the external boundary of the pipe. The optimization problem consists of finding the shape for the external boundary keeping the internal one fixed and minimizing the total area. The 4 design variables $V_{i}, i=1 \ldots 4$, shown in Figure 1 correspond with the coordinates of the points that define the external boundary.

The maximum von-Mises stresses along the boundary of the model have been restricted to $2,0 \times 10^{6}$.

The area of the analytical solution for this optimization problem ( $1 / 4$ of cross section) is $A_{\text {opt }}=69,725903$. This corresponds to an external radius $R_{O}=10,666666$.

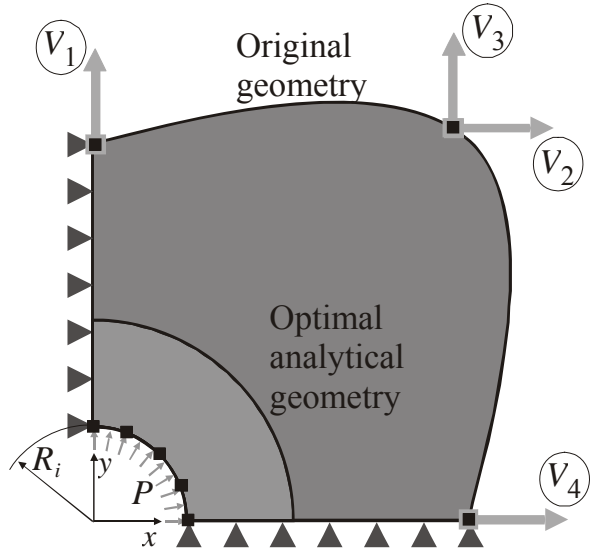

Plane strain

$E=10.1 \cdot 10^{6}$

$v=0.3$

$P=0.9 \cdot 10^{6}$

$R_{i}=5$

Fig. 1. Pipe cross section. Analysis data, original model, optimal analytical solution and design variables

\subsection{Evolutive algorithm}

For these numerical analyses we have used the Differential Evolution (DE) algorithm. DE is an evolutionary algorithm that has shown a robust performance yielding good results, even when applied to very different types of problems. DE was developed by Storn and Price in 1995 (see [2]). Its key idea is the differential operator, which serves the same purpose as the crossover parameter in a standard genetic algorithm, namely to exchange information between parents when creating offspring. We have used the DE1 classical version of the Differential Evolution proposed by Storn and Price.

The initial values of the design variables and their allowed data range and constraints for the Pipe cross section problem are shown in Table I.

Table 1. Pipe cross section. Values of design variables

\begin{tabular}{cccl}
\hline $\begin{array}{l}\text { Design } \\
\text { variable }\end{array}$ & $\begin{array}{c}\text { Initial } \\
\text { value }\end{array}$ & Range & Constraints \\
\hline$V_{1}$ & 20 & {$[5.2-50.0]$} & \\
$V_{2}$ & 19 & {$[4.0-50.0]$} & \\
$V_{3}$ & 19 & {$[4.0-50.0]$} & $V_{3}<V_{1}-0.5$ \\
$V_{4}$ & 20 & {$[5.2-50.0]$} & $V_{4}<V_{2}+0.5$ \\
\hline
\end{tabular}

Observe that we have used constraint equations between the values of the design variables in order to minimize the production of geometrically unfeasible individuals.

\subsection{Gradient-based algorithm}

The deterministic optimization algorithm considered in this work defines the new values of the design variables for a new design using the results of the numerical analyses associated to the behavior of the existing design, and their sensitivity analysis. The methodology used in this paper corresponds to the algorithm developed by Navarrina [3]. Once the $k$-th design has been analyzed, the values for the design variables for the next design are defined as:

$$
\mathbf{x}_{k+1}=\mathbf{x}_{k}+\theta_{k} \mathbf{s}_{k}
$$

where $\mathbf{x}_{k}$ is the vector containing the values of the design variables for the $k$-th geometry, $\mathbf{x}_{k+1}$ is the vector corresponding to the next geometry, $\mathbf{s}_{k}$ is a unit vector defining the direction of change in the space of the design variables and $\theta_{k}$ is a scaling factor in this direction.

The algorithm computes the direction of change $\mathbf{s}_{k}$ by using a SIMPLEX method with information coming from an exact first order sensitivity analysis of the OF and the constraints. Next, the scaling factor $\theta_{k}$ is computed by performing a line search minimization using second order directional sensitivity analysis.

\subsection{H-Adaptive Finite Element Analysis}

We have used an $h$-adaptive finite element analysis code to obtain the values of the objective function and the degree of satisfaction of the constraints for each of the different geometrical configurations proposed by the optimization algorithms. The use of the $h$-adaptive strategy ensures the 
quality of the analysis providing FE solutions with a relative estimated discretization error in energy norm $\gamma$ lower than that specified by the analyst. The program uses the Zienkiewicz-Zhu error estimator in energy norm [4] to guide the $h$-adaptive analysis. Hence, the following expression is used to evaluate the error estimation in energy norm for each element, $\eta_{\mathrm{e}}$ :

$$
\eta_{e}^{2}=\int_{\Omega_{e}}\left(\boldsymbol{\sigma}^{*}-\boldsymbol{\sigma}_{h}\right)^{T} \mathbf{D}^{-1}\left(\boldsymbol{\sigma}^{*}-\boldsymbol{\sigma}_{h}\right) d \Omega
$$

where $\boldsymbol{\sigma}_{h}$ is the stress field directly obtained from the FE analysis, $\boldsymbol{\sigma}^{*}$ is a recovered stress field, $\mathbf{D}$ relates strains with stresses as $\boldsymbol{\sigma}=\mathbf{D} \boldsymbol{\varepsilon}$ and $\Omega_{e}$ is the domain of element $e$.

In the numerical examples, triangular elements have been used due to their big flexibility when generating adapted unstructured meshes. Quadratic (6 noded) instead of linear (3 noded) triangular elements have been used in order to improve accuracy. We have used the global least squares smoothing technique [4] to obtain the recovered stress field $\boldsymbol{\sigma}^{*}$ required by the Zienkiewicz-Zhu error estimator. Other recovery techniques like the Superconvergent Patch Recovery technique (SPR) by Zienkiewicz and Zhu[5] or any other improvement of this recovery technique could also be used [6-8].

Cubic B-splines [9] have been used to define each of the geometrical configurations in terms of the coordinates of some definition points (parameterization).

In order to see the effect of the amount of discretization error over the behavior of the optimization algorithms (both evolutionary and deterministic) 6 different situations corresponding to different prescribed error levels have been studied. The maximum error values prescribed for the first 5 analyses were $1 \%, 2.5 \%, 5 \%, 10 \%$ and $20 \%$. In the $6^{\text {th }}$ case a $\gamma<100 \%$ tolerance has been specified, which in practical terms implies that the accuracy of the solution is not controlled and the number of elements is only depending on geometrical criteria (see examples in Figure 2).
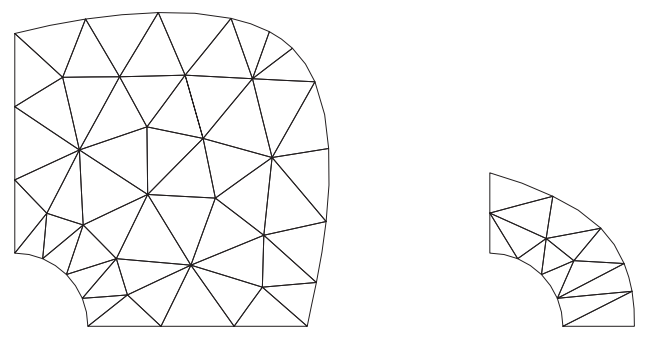

Fig. 2. Examples of initial meshes

\subsection{Solution using the evolutionary algorithm. Influence of the discretization error}

Figure 3 shows the effect of the prescribed maximum estimated relative error in energy norm $\gamma$ over the evolution of the area of the pipe cross section. It can be observed that, at least for this problem, the global aspect of the results for all the situations is quite similar.

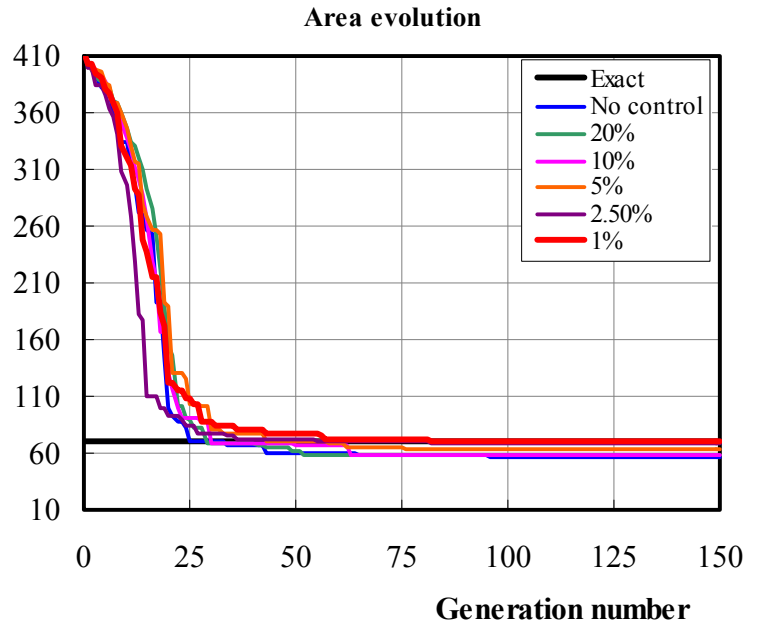

Fig. 3. Influence of $\gamma$ over the evolution of the objective function

However, as shown in Figure 4, the effect of $\gamma$ over the evolution of the optimization process is especially significant if we compare the final results obtained with each different degree of the discretization error. Figure 4 shows, in relative terms, the evolution of the difference between the area provided by the best individual obtained up to each generation and the area of the optimal analytical solution. The graph shows that the final solution associated to each analysis is significantly different to the analytical solution for the cases with higher values of the prescribed error $\gamma$, but approaches to the exact solution for the lower values of $\gamma$.

Figure 5 represents the effect of $\gamma$ over the final solution provided by the optimization algorithm. It can be clearly observed that for high values of $\gamma$ the algorithm converges to shapes that are quite different to the optimal analytical solution. However, the final solution provided by the optimization process approaches to the analytical solution for decreasing values of $\gamma$. Observe that the difference between the exact solution and that obtained for $\gamma=1 \%$ is only $0.46 \%$. In any case, one should take into account that the optimal analytical external boundary (circular shape) cannot be exactly obtained because the $B$-splines used to define de boundary are unable to exactly reproduce an exact circular shape.

The finite element method usually underestimates the maximum stress value. The level of this underestimation of stresses increases with the size of the elements used in the analysis. In order to quantify the quality of the solutions obtained with the different degrees of discretization error, we have performed a full $h$-adaptive analysis requiring a final estimated error in energy norm $\gamma<0.3 \%$ over each of the geometries displayed in Figure 5. This has produced much more accurate approximations of the stress distributions and, therefore, a much more accurate evaluation of the degree of satisfaction of the stress constraints. Figure 6 displays the difference between the maximum von Mises stresses finally obtained for each design and the maximum allowed value for this magnitude. It is clearly shown that the obtained optimal solutions underestimate the maximum value of the von Mises stress. Hence, the accurate evaluation of these designs reveals that they would severely exceed this magnitude. The same figure also shows the difference between the final area obtained for each value of $\gamma$ and the area 
of the analytical solution. It can be clearly observed that both graphs are closely related. Basically, due to the underestimation of the maximum von Mises stress the optimization algorithm reduces the area until this value reaches the specified limit. Hence, the optimization algorithm provides solutions with a lower area than those that would be obtained with an accurate evaluation of the maximum von Mises stress. Observe that the solution obtained with $\gamma \leq$ $1 \%$ is almost identical to the analytical solution, with a difference in areas of only $+0.46 \%$ and exceeding the allowable stresses by only $0.68 \%$.

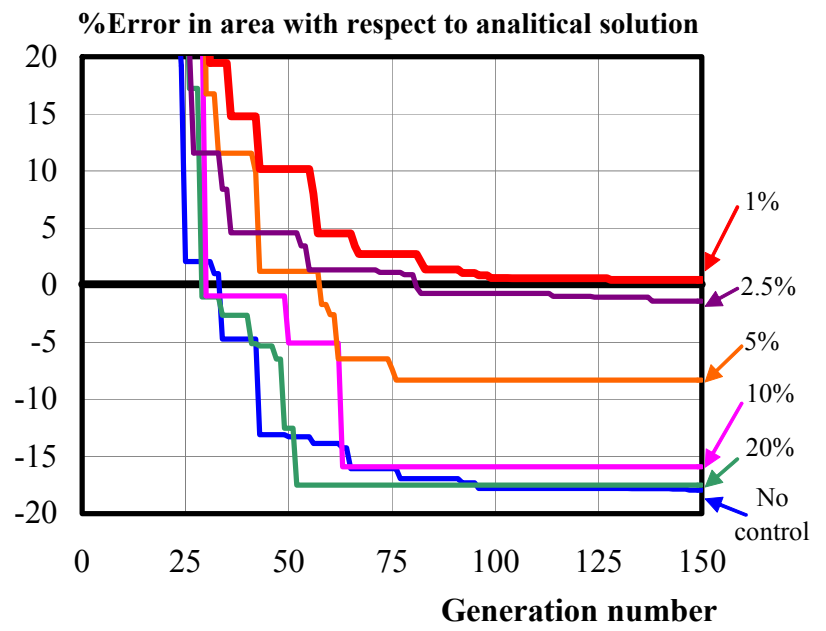

Fig. 4. Influence of $\gamma$ over the evolution of the error in the objective function with respect to the analytical solution
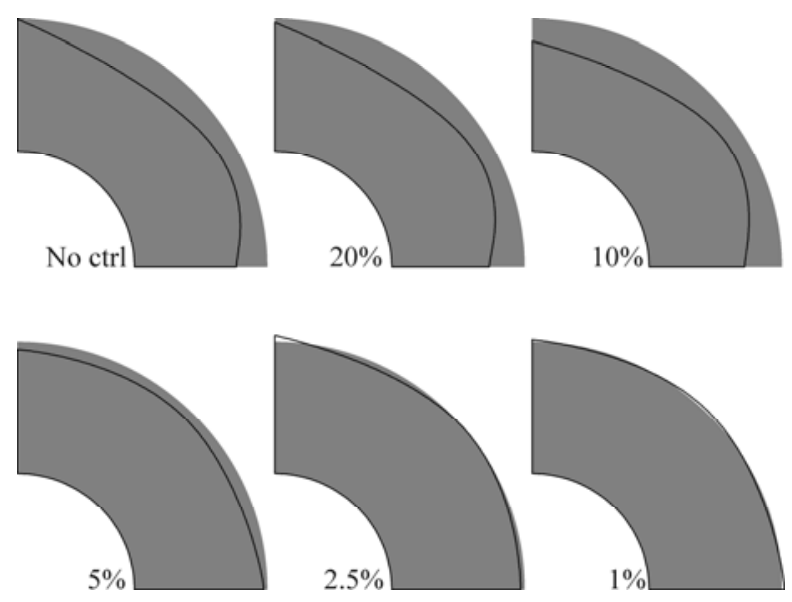

Fig. 5. Influence of $\gamma$ over the optimal solution found (black contour) and comparison with optimal analytical solution (shaded area)

\subsection{Solution using the gradient-based determi- nistic algorithm. Influence of the discretization error}

Figure 7 shows a comparison between the convergence curves obtained with the different levels of prescribed discretization error for the case of the gradient-based deterministic algorithm. First, it can be seen that in all cases the convergence is much faster than with the evolutionary algorithm. Nevertheless, it can also been seen that after an initial quick drop of the OF, its evolution is different depending on the amount of the allowed discretization error.

During the first 10 iterations the convergence is not depending on the level of discretization error. This is because in these initial iterations the obtained designs are far away from the optimal one and the corresponding stress values are still far away from the restricted ones. Therefore, the constraints are not yet active and thus the process is still independent of the degree of accuracy in the evaluation of the constraints.

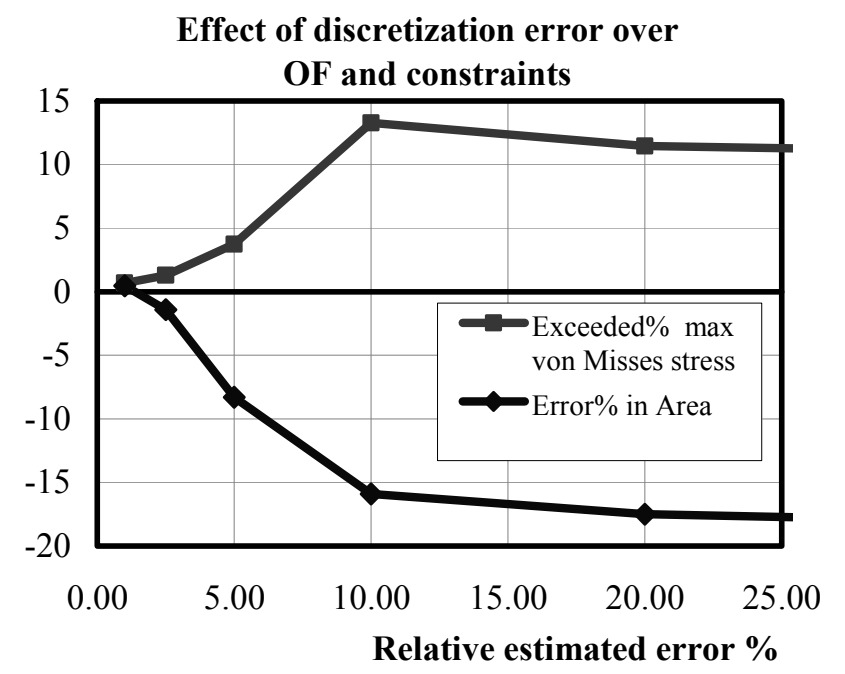

Fig. 6. Influence of $\gamma$ over the accuracy of the objective function and the degree of satisfaction of stress constraint equations

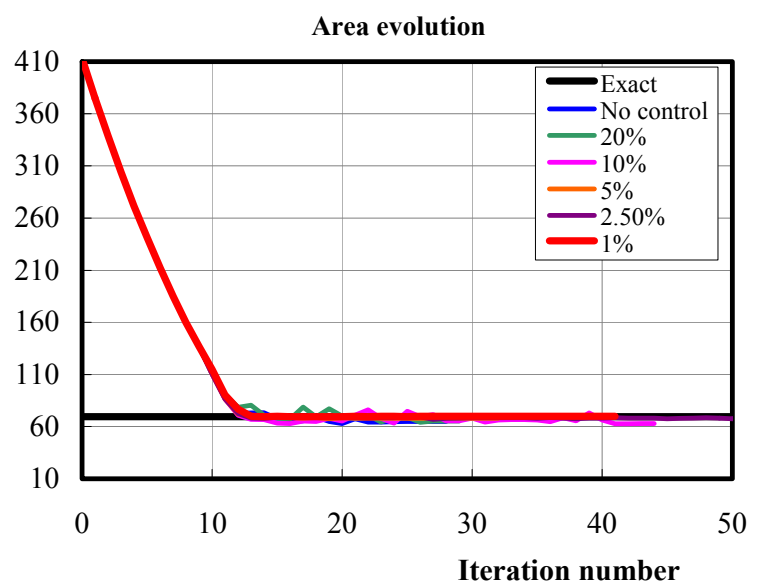

Fig. 7. Deterministic algorithm. Influence of $\gamma$ over the evolution of the objective function

Fig. 8 shows the detailed evolution of the OF after the initial iterations. In this case, the evolution of the relative differences between the cross sectional area obtained at each iteration and the optimal analytical one is represented. It can be seen how a quick convergence to a practically exact value is obtained when $\gamma$ is fixed to $1 \%$. On the other hand, when higher values of $\gamma$ are employed, the evolution of the OF shows big oscillations and the process stops with final designs that, in fact, are not feasible because a more accu- 
rate analysis also shows too high values of the von Mises stress. The main reason of all this behavior is the lack of reliability of the obtained values for the von Mises stress that drive all the optimization process. These values change every time the mesh is modified, introducing a lot of "noise" in the convergence of the process and in the lack of reliability of the final design. This noise would not appear if a single mesh, conveniently adapted to each geometry, had been used. In this case, this noise wouldn't be present and wouldn't produce oscillations, but the final optimum design wouldn't be reliable because the degree of reliability of the computed von Mises stress would be very low.

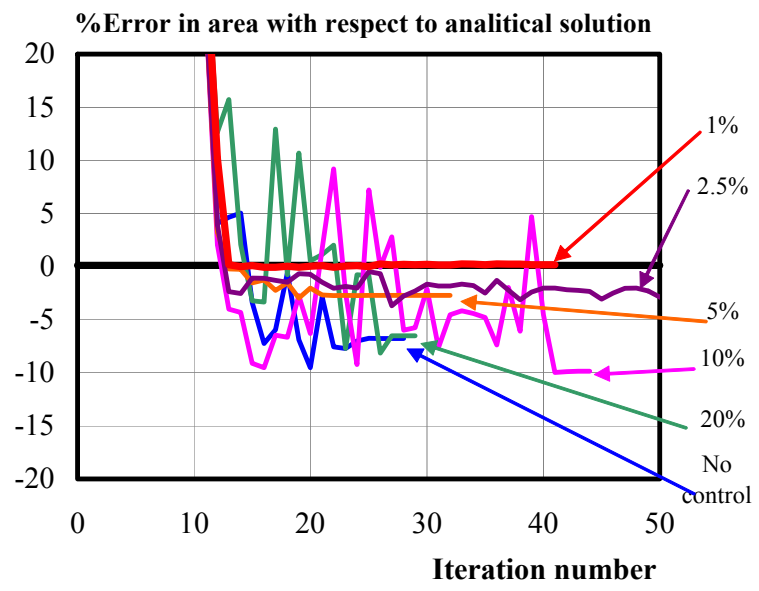

Fig. 8. Deterministic algorithm. Influence of $\gamma$ over the evolution of the difference between the obtained objective function and the analytical solution
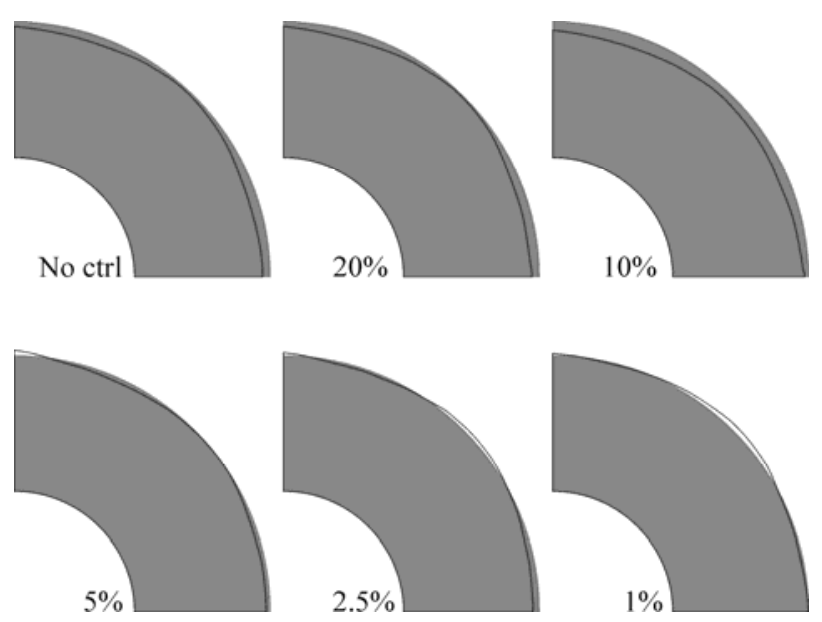

Fig. 9. Deterministic algorithm. Influence of $\gamma$ over the optimal solution found (black contour) and comparison with optimal analytical solution (shaded area).

As in the case of the evolutionary algorithm, and in order to quantify the quality of the solutions obtained with the different degrees of discretization error, a full $h$-adaptive analysis requiring a final estimated error in energy norm $\gamma<0.3 \%$ was performed over each of the geometries displayed in Fig. 9. These analyses produced much more accurate approximations of the stress distributions and, thus, much more accurate evaluation of the degree of satisfaction of the stress constraints. Fig.10 displays the difference between the maximum von Mises stresses finally obtained for each design and the maximum allowed value for this magnitude. The results are similar to those previously shown for the evolutionary algorithm: the optimal solutions provided by the optimization process underestimate the maximum value of the von Mises stress that would be obtained with more accurate FE models. Solutions with a lower area than those that would be obtained with an accurate evaluation of the maximum von Mises stress are, thus, obtained. However, the results obtained in this case are more accurate than those obtained with the evolutionary algorithm. The solution obtained with $\gamma \leq 1 \%$ is again quite close to the analytical solution, with a difference in areas of only $+0.061 \%$ and exceeding the allowable stresses by only $0.33 \%$.

Effect of discretization error over $O F$ and constraints

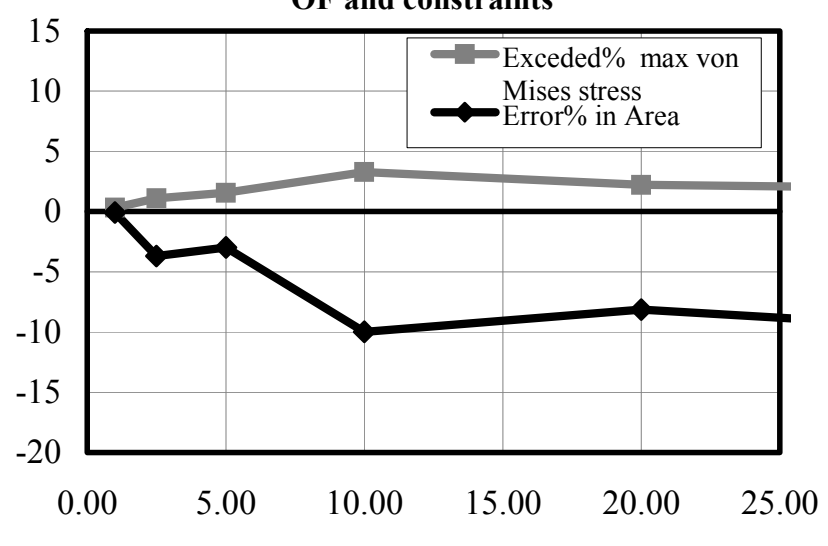

Relative estimated error \%

Fig. 10. Deterministic algorithm. Influence of $\gamma$ over the accuracy of the objective function and the degree of satisfaction of stress constraint equations.

\section{Error-control strategies for evolutionary algorithms}

The numerical results previously exposed have clearly shown the importance of the accuracy of the FE analysis on the final results of both types of optimization algorithms. Mesh refinement in FE analyses is directly associated to error control techniques. In fact, to minimize the total amount of calculations one should preferably use some kind of adaptive analysis technique such us the automatic $h$-adaptive mesh refinement technique previously mentioned as they tend to produce the solution with the prescribed accuracy at the lowest computational cost. The use of such techniques is especially critical when evolutionary algorithms are used due to the large amount of different geometrical configurations to be analyzed. In this case, and in order to reduce the computational cost associated to the creation of an adapted mesh for each of the individuals to be analyzed, we propose the use of the $h$-adaptive strategy for the analysis of generations of individuals described in [1]. This technique has been used in the numerical example presented in the following section. The origin of this technique, and its application to deterministic optimization algorithms, can be seen in references [10-12]. The work in [1] presents a strategy that allows generating an adapted mesh for each individual of a generation without the necessity of performing a full adaptive remeshing procedure for each of them. It 
makes use of sensitivity analysis of all magnitudes related with adaptive remeshing (location of nodes, error estimation, etc.) in a reference geometry with respect to the design variables. This sensitivity analysis is then used to project the results from the reference geometry to all other geometrical configurations. This information allows generating an appropriate $h$-adapted mesh for each geometry, thus avoiding the full $h$-adaptive loop over each individual.

However, the results previously presented have also shown that the accuracy of the analyses, prescribed by $\gamma$, does not significantly modify the early stages of the optimization process. This suggests the use of a very simple error control technique to reduce the total amount of calculations involved in the optimization process. The error control technique consists of using high values of $\gamma$ at the early stages of the optimization process and to successively decrease this value with the advance of the optimization process.

\section{Numerical example: Gravity dam}

The gravity dam represented in Figure 9 was analyzed. Both dead weight and water hydrostatic pressure were considered in the analysis. The model does not include any sharp reentrant corners. The shape optimization problem consists of finding the best shape for the internal boundary, keeping fixed the external one. This minimizes the cross sectional area of the gravity dam. A total of 7 points were used to define the internal boundary. As indicated in Fig. 10, the coordinates of 5 of these points were considered as the design variables for this problem. The maximum von-Mises stresses along the boundary of the model have been restricted to $2,75 \cdot 10^{6} \mathrm{~Pa}$.

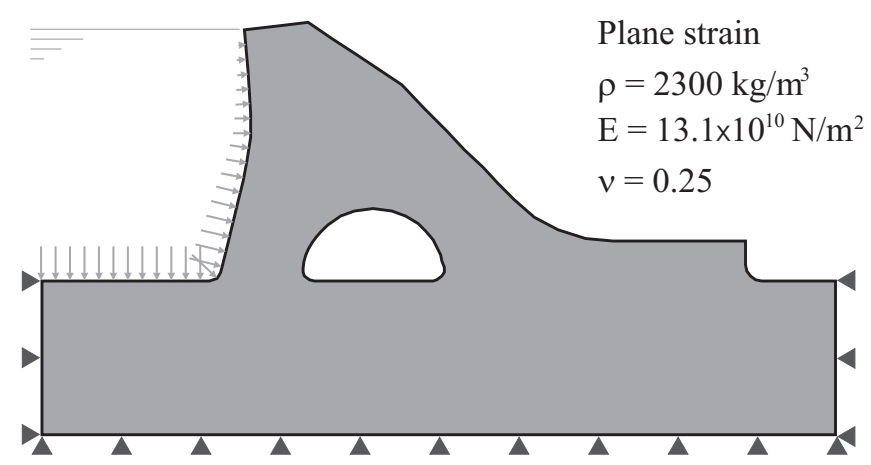

Fig. 9. Gravity dam. Analysis model

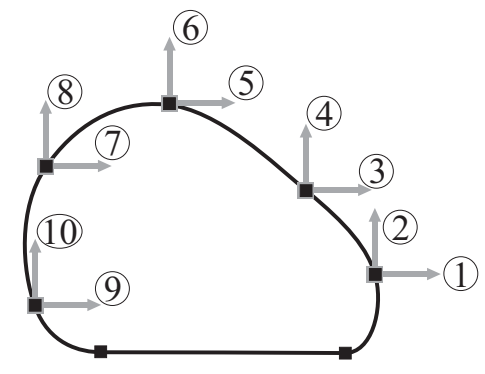

Fig. 10. Gravity dam. Design variables
30 individuals per generation were used in the analysis. The analysis ran for a total of 100 generations. A maximum estimated relative error in energy norm $\gamma=2.5 \%$ was required for the analysis of the final solution. We specified a geometrical variation of the estimated error in energy norm from $\gamma=20 \%$ in the first generation to $\gamma=2.5 \%$ in the last one (see Fig. 11) to reduce the computational cost associated to the early stages of the optimization process.

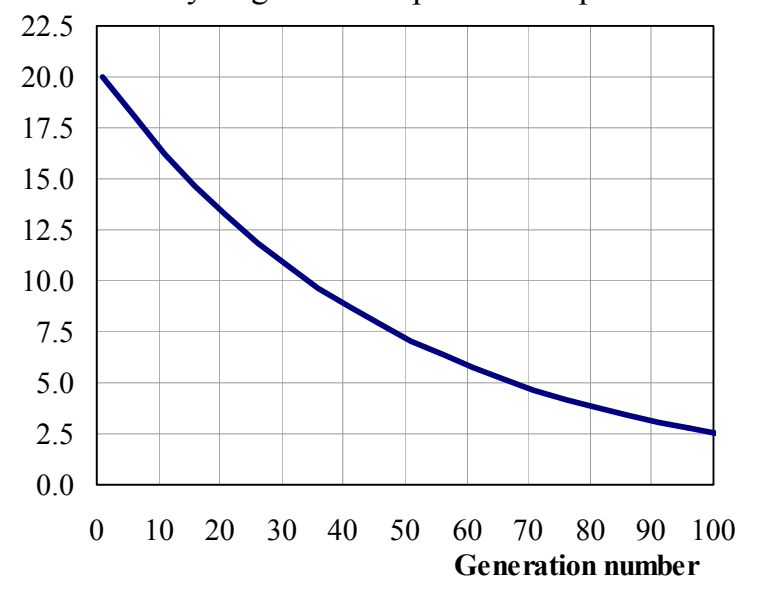

Fig. 11. Evolution of the prescribed value of $\gamma$.

In this test case, the use of the error projection technique previously described [1] provided the appropriate mesh for the FE analysis of $95 \%$ of the individuals considered in the optimization process, further reducing the computational cost of the optimization.

Fig. 12 shows the evolution of the objective function (area) during the optimization process. Fig. 13 clearly shows the difference between the original and the optimized designs. After the analysis of 100 generations, a previous test, where the maximum estimated relative error in energy norm was prescribed as $\gamma=3 \%$ for the whole optimization process, provided an area whose difference with respect to the final result shown in Fig. 12 is only $0.7 \%$. As expected, the use of the previously described strategy where a variable value $\gamma$ is prescribed, does not put the accuracy of the optimization process at risk.

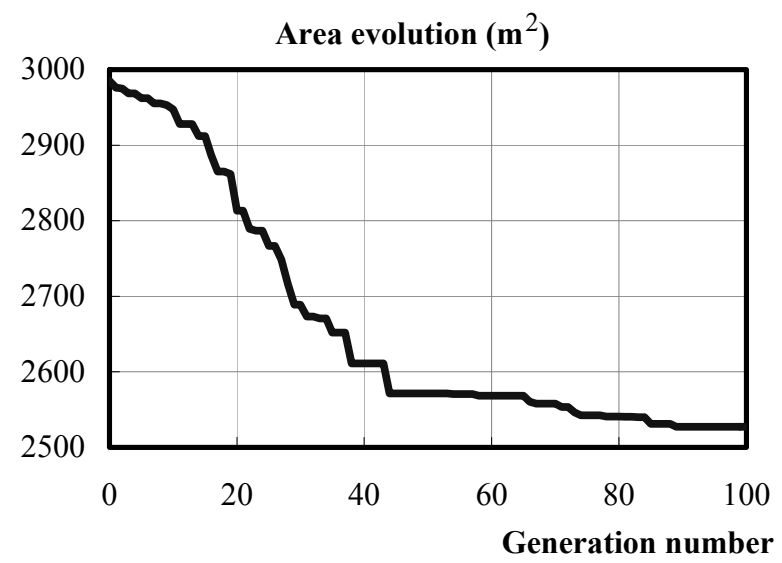

Fig. 12. Gravity dam. Area evolution 


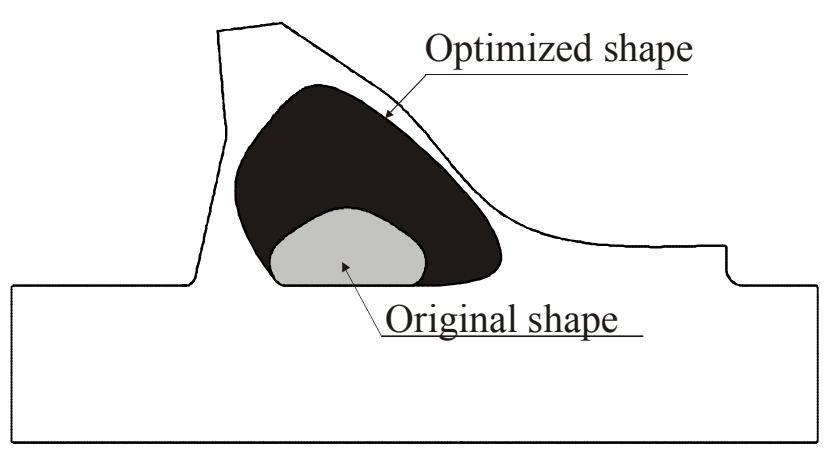

Fig. 13. Gravity dam. Optimized geometry versus original geometry

\section{Conclusions}

This paper has shown that a minimum quality of the results used to drive structural shape optimization processes must be ensured; otherwise the process will not converge to the optimal solution, providing solutions that could notably violate the satisfaction of the constraint equations. Therefore, the optimization processes that make use of the finite element method to evaluate the objective function and the degree of satisfaction of constraints require for the use of FE model refinement analysis techniques to ensure the quality of the results.

Due to the high number of individuals to be analyzed, the computational cost associated to the use of adaptive analysis techniques can be critical when evolutionary algorithms are considered. For this kind of optimization algorithms we propose the use of the technique for the $h$-adaptive analysis of generations of individuals presented in [1]. This technique uses the projection of the estimated discretization error in energy norm to obtain the meshes required for the analysis of the individuals considered in each generation. The results presented show the efficiency of this technique which in the vast majority of cases avoids the full adaptive remeshing process.

Preliminary results have shown that the maximum discretization error level imposed over the FE analyses at the beginning of the optimization has no significant effect over the final results of the optimization process. Hence, we have proposed the use of a technique where the maximum discretization error level decreases with the progress of the optimization process. This technique considerably reduces the computational cost associated to the analysis of the first generations of individuals without affecting the accuracy of the results corresponding to the last steps of the optimization process.

\section{Acknowledgement}

The authors have been sponsored by the Spanish Ministerio de Ciencia e Innovación through the projects DPI2008-05250 (first author) and DPI2007-66773-C0201 (second and third authors). The second and third authors have also been sponsored by the Generalitat Valenciana and the Universidad Politécnica de Valencia.

\section{References}

1. G. Bugeda, J. J. Ródenas, E. Oñate, An integration of a low cost adaptive remeshing strategy in the solution of structural shape optimization problems using evolutionary methods, Computers \& Structures, 86, 1563-1578 (2008).

2. R. Storn, K. Price. Differential evolution - A simple and efficient adaptive scheme for global optimization over continuous space. International Computer Science Institute, Berkeley, CA, USA (1995). Available:

ftp://ftp.icsi.berkeley.edu/pub/techreports/1995/tr-95012.pdf

3. F. Navarrina, Una metodología general para optimización structural en diseño assitido por ordenador, Phd Thesis, Universitat Politècnica de Catalunya, in spanish (1987).

4. O. C. Zienkiewicz, J. Z. Zhu, A simple error estimator and adaptive procedure for practical engineering analysis, International Journal for Numerical Methods in Engineering, 24, 337-357 (1987).

5. O. C. Zienkiewicz, J. Z. Zhu, The superconvergent patch recovery and A posteriori error estimates. Part 1: The recovery technique, International Journal for Numerical Methods in Engineering, 33, 1331-1364 (1992).

6. N. E. Wiberg, F. Abdulwahab, S. Ziukas, Enhanced superconvergent patch recovery incorporating equilibrium and boundary conditions, International Journal for $\mathrm{Nu}-$ merical Methods in Engineering, 37, 3417-3440 (1994).

7. J. J. Ródenas, M. Tur, F. J. Fuenmayor, A. Vercher, Improvement of the superconvergent patch recovery technique by the use of constraint equations: the SPR-C technique, International Journal for Numerical Methods in Engineering, 70, 705-727 (2007).

8. P. Díez, J. J. Ródenas, O. C. Zienkiewicz, Equilibrated patch recovery error estimates: simple and accurate upper bounds of the error, International Journal for Numerical Methods in Engineering, 69, 2075-2098 (2007).

9. I. D. Faux, M. J. Pratt, Computational Geometry for Design and Manufacture. [5th. reprint.] ed.Chichester: Ellis Horwood (1987).

10. G. Bugeda, Oliver, A general methodology for structural shape optimization problems using automatic adaptive remeshing, International Journal for Numerical Methods in Engineering, 36, 3161-3185 (1993).

11. G. Bugeda, E. Oñate, A methodology for adaptive mesh refinement in optimum shape design problems, Computing Systems in Engineering, 5, 91-102 (1994).

12. F. J. Fuenmayor, J. L. Oliver, J. J. Ródenas, Extension of the Zienkiewicz-Zhu error estimator to shape sensitivity analysis, International Journal for Numerical Methods in Engineering, 40, 1413-1433 (1997). 